\title{
CONDUCTION OF HEAT FROM LOCAL SOURCES IN A MEDIUM GENERATING OR ABSORBING HEAT
}

\author{
by S. PATERSON
}

(Received 20th December, 1952)

\section{Abstract}

An infinite or semi-infinite medium, in which heat is generated or absorbed at a rate proportional to the temperature, is placed at temperature zero in contact with a perfect conductor of finite heat capacity at a higher temperature. Expressions are derived for the subsequent behaviour in linear and spherical cases, and applications suggested.

\section{(1) Linear Case}

A semi-infinite medium $(x>0)$ of diffusivity $\kappa$, thermal conductivity $k$ and temperature zero is placed in contact over the face $x=0$ with a well-stirred fluid or perfect conductor of thermal capacity $c^{\prime}$ per unit area, at temperature $T_{\mathbf{0}}$. The temperature $T^{\prime}$ of the fluid is assumed equal at all subsequent times $t$ to the surface temperature of the medium, in which heat is generated or absorbed at a rate proportional to the temperature $T(x, t)$.

Then

$$
\begin{aligned}
\frac{\partial T}{\partial t} & =\kappa \frac{\partial^{2} T}{\partial x^{2}}+G T, \quad x>0, \quad t>0, \\
T & =0, \quad x>0, \quad t=0 \\
T & =T^{\prime}, \quad x=0, \quad t>0, \\
k \partial T / \partial x & =c^{\prime} d T^{\prime} / d t, \quad x=0, t>0, \\
T^{\prime} & =T_{0}, \quad t=0,
\end{aligned}
$$

where $G$ is a constant, which may be positive or negative.

For convenience, set $\kappa t \equiv \tau, T / T_{0} \equiv u, T^{\prime} / T_{0} \equiv u^{\prime}, G / \kappa \equiv H$, and $k / \kappa c^{\prime} \equiv w$. Then the equations become

$$
\left.\begin{array}{rl}
\frac{\partial u}{\partial \tau} & =\frac{\partial^{2} u}{\partial x^{2}}+H u \\
u & =0, \quad \tau=0 \\
u & =u^{\prime}, \quad x=0 \\
w \partial u / \partial x & =d u^{\prime} / d \tau, \quad x=0 \\
u^{\prime} & =1, \quad \tau=0
\end{array}\right\}
$$

If $\bar{u} \equiv L(u) \equiv \int_{0}^{\infty} e^{-p \tau} u d \tau$ denotes,the Laplace transform of $u$, these equations transform into

$$
\left.\begin{array}{c}
\frac{d^{2} \bar{u}}{d x^{2}}=(p-H) \bar{u}, \\
w \frac{d \bar{u}}{d x}=p \bar{\imath}-1, \quad x=0
\end{array}\right\}
$$

whose solution, finite at $x=\infty$, is

$$
\bar{u}=\frac{e^{-\alpha x}}{p+\alpha w}
$$


where

$$
\alpha \equiv \sqrt{p-\bar{H}}
$$

$u$ is then determined by the inverse transform

$$
u=L^{-1}(\bar{u})=\frac{1}{2 \pi i} \int_{L} \frac{e^{p_{\tau}-\alpha x}}{p+\alpha w} d p,
$$

where $L$ passes from $I(p)=-\infty$ to $I(p)=+\infty$ and to the right of the zeros of $p+w \alpha$. The substitution

$$
p+w \alpha \equiv \lambda
$$

enables us to write

$$
u=e^{\frac{w}{2}(x+w \tau)}[J-K]
$$

where

$$
\begin{aligned}
& J \equiv \frac{1}{2 \pi i} \int_{M} e^{\lambda \tau-(x+w \tau)} \sqrt{\lambda+\frac{w^{2}}{4}-H} \frac{d \lambda}{\lambda}, \ldots \ldots \ldots \ldots \ldots . . . \\
& K \equiv \frac{w}{2 \pi i} \int_{M} e^{\lambda \tau-(x+w \tau)} \sqrt{\lambda+\frac{w^{2}}{4}-H} \frac{d \lambda}{\lambda \sqrt{\lambda+\frac{w^{2}}{4}-H}},
\end{aligned}
$$

and $M$ is the corresponding contour. We may denote $x+w \tau$ by $y$ and $\frac{w^{2}}{4}-H$ by $g^{2}$. Then, by the superposition theorem, $J=\int_{0}^{\tau} \phi(s) d s$ and $K=\int_{0}^{\tau} \psi(s) d s$, where

$$
\begin{aligned}
\phi(s) & =\frac{1}{2 \pi i} \int_{M} e^{\lambda s-y^{\sqrt{\lambda+g^{2}}}} d \lambda \\
& =\frac{e^{-g^{2} s}}{2 \pi i} \int_{M} e^{\lambda s-y^{\sqrt{\lambda}}} d \lambda \\
& =\frac{y}{2 \sqrt{\pi s^{3}}} \cdot e^{-g^{2} s-y^{2} / 4 s}, \ldots
\end{aligned}
$$

and similarly

$$
\begin{aligned}
\psi(s) & =\frac{w e^{-g^{2} s}}{4 \pi i} \int_{M} e^{\lambda s-y^{\sqrt{ }} \bar{\lambda}} d \lambda / \sqrt{\lambda} \\
& =\frac{w}{2 \sqrt{\pi s}} \cdot e^{-g^{2} s-y^{2} / 4 s} \cdot \ldots \ldots
\end{aligned}
$$

Hence, finally, from (8), (11), (12),

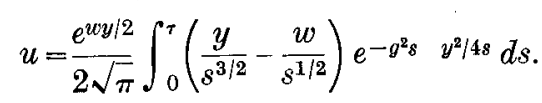

It can be confirmed by direct substitution that (13) satisfies the conditions of the problem.

An alternative form of the solution, which is more convenient if $g^{2}>0$, can be obtained, either from (13) by using two relations given by Horenstein,(1), or more directly from the transform (4). For, if $p \equiv \mu^{2}+H$, (6) becomes

$$
u=\frac{1}{\pi i} \int_{M} \exp \left\{\left(\mu^{2}+H\right) \tau-\mu x\right\} \frac{\mu d \mu}{\mu^{2}+w \mu+H}
$$

$\mathbf{L}$ G.M.A. 
By resolving into partial fractions, we easily deduce

$$
\begin{aligned}
u=\frac{1}{2 g}\left[\left(g-\frac{w}{2}\right) e^{-v\left(g-\frac{w}{2}\right)} \operatorname{erfc}\left(\frac{y}{2 \sqrt{\tau}}-g \sqrt{\tau}\right)\right. \\
\left.+\left(g+\frac{w}{2}\right) e^{y\left(g+\frac{w}{2}\right)} \operatorname{erfc}\left(\frac{y}{2 \sqrt{\tau}}+g \sqrt{\tau}\right)\right] .
\end{aligned}
$$

When there is no generation of heat, $g=\frac{w}{2}$, and (15) reduces to

$$
u=e^{w y} \operatorname{erfc}\left(\frac{y+w \tau}{2 \sqrt{\tau}}\right)
$$

The same expression can be derived with rather more trouble from (13), or, of course, directly from (4), with $\alpha=\sqrt{p}$.

A series solution suitable for large values of $x / 2 \sqrt{\tau}$ can be obtained as follows. By (6),

where

$$
u=e^{\boldsymbol{B} \tau} \cdot v \text {, }
$$

$$
\bar{v}=\frac{e^{-x} \sqrt{\bar{p}}}{p+w \sqrt{p}+H}
$$

Writing $q$ for $\sqrt{p}$, and expanding in negative powers of $q$, we have

where

$$
\bar{v}=\frac{e^{-x q}}{q^{2}}\left(1+B_{1} q^{-1}+B_{2} q^{-2}+\ldots\right)
$$

Hence,

$$
\begin{aligned}
& B_{1}=-w, \\
& B_{2}=w^{2}-H, \\
& B_{3}=2 H w-w^{3}, \\
& B_{4}=w^{4}-3 H w^{2}+H^{2},
\end{aligned}
$$

if, in Hartree's (2) notation,

$$
u=e^{H \tau}\left[\operatorname{erfc} \frac{x}{2 \sqrt{\tau}}+\sum_{1}^{\infty} B_{n}(2 \sqrt{\tau})^{n} i^{n} \operatorname{erfc} \frac{x}{2 \sqrt{\tau}}\right]
$$

and

Since, when $z$ is large,

$$
i^{n} \operatorname{erfc} z \equiv \int_{z}^{\infty} i^{n-1} \operatorname{erfc} z d z
$$

$$
i^{0} \operatorname{erfc} z \equiv \operatorname{erfc} z \text {. }
$$

$$
i^{n} \operatorname{erfc} z \sim \frac{2}{\sqrt{\pi}}(2 z)^{-(n+1)} e^{-z^{2}},
$$

the series (21) converges rapidly for $x / 2 \sqrt{\tau} \gg 1$. When $x=0,(21)$ reduces to

(22) converges rapidly for small $\tau$.

$$
\begin{aligned}
u^{\prime} & =e^{\boldsymbol{H} \tau}\left[1+\sum_{1}^{\infty} \frac{B_{n} \tau^{n / 2}}{\Gamma\left(\frac{n}{2}+1\right)}\right] \\
& \equiv e^{B \tau}\left[1-2 w \sqrt{\frac{\tau}{\pi}}+\left(w^{2}-H\right) \tau+\ldots\right] .
\end{aligned}
$$

In what follows, we shall assume $T_{0}>0, H>0$. Then it is clear from (22) that the surface temperature commences by falling, irrespective of the value of $H$. From physical considerations, however, it must subsequently rise again, in consequence of the generation of heat, if 
$H>0$. Although it appears difficult to define the turning-point in general terms, the behaviour may be illustrated by a special case, corresponding to $w^{2}=4 H$. For then $g=0$ and the surface temperature is therefore, by (15),

where

$$
u(0)=e^{2 \xi^{2}}\left\{\left(1+2 \xi^{2}\right) \operatorname{erfc} \xi-\frac{2 \xi}{\sqrt{\pi}} e^{-\xi^{2}}\right\},
$$

The turning-value is defined by

$$
0=\frac{d u(0)}{d \xi}=4 e^{2 \xi^{2}}\left\{2 \xi\left(1+\xi^{2}\right) \operatorname{erfc} \xi-\frac{1}{\sqrt{\pi}}\left(1+2 \xi^{2}\right) e^{-\xi^{2}}\right\}
$$

which has a single root $\sim 0.847$.

As an example, let us suppose a cold explosive gas to be brought in contact with a hot metallic foil. Heat will be generated in the gas according to an Aarhenius function of temperature, which we may represent very roughly by the linear equation

if $G$ is defined by

$$
(\partial T / \partial t)_{\text {react. }}=G T,
$$

$$
G T_{0}=A e^{-E / R T_{0}}
$$

where $A \sim 10^{12}{ }^{\circ} \mathrm{K} / s$. Provided the condition $w^{2}=4 H$ is satisfied, we may then expect the surface temperature to commence rising after a time $t_{c}$ defined by $\xi \sim 0 \cdot 85$, that is, by

$$
G t_{0} \sim 0.7 \text {. }
$$

By (26), this reduces to

$$
t_{c} \sim 0.7 \times 10^{-12} T_{\mathbf{0}} e^{E / R T_{\mathbf{0}}}
$$

Assuming $E \sim 40,000 \mathrm{cal} . / \mathrm{mole}$, we have the following variation of $t_{c}$, which may be identified with the lag time for ignition.

$$
\begin{array}{lccccccc}
T_{0}\left({ }^{\circ} \mathrm{K}\right) & 5000 & 4000 & 3000 & 2000 & 1000 & 700 & 500 \\
t_{c} \text { (sec.) } & 2 \times 10^{-7} & 4 \times 10^{-7} & 2 \times 10^{-6} & 3 \times 10^{-5} & 3 \times 10^{-1} & 10^{3} & 8 \times 10^{7}
\end{array}
$$

It is clear that ignition in any normal sense requires a foil temperature of the order of $1000^{\circ} \mathrm{K}$, which is a physically reasonable level. Since $G$ must evidently be $\sim 1$, the condition $w^{2}=4 H$ implies $w \sim 2 / \sqrt{\kappa} \sim 1$, which corresponds to a foil thickness of the order of $10^{-3} \mathrm{~cm}$. Although this example is a rather crude one, it does illustrate the possibility of a useful application of the present analysis. No doubt this would be more profitable in the case of condensed explosives, where convective effects are absent.

(2) Spherical Case

If $R$ denotes a radial co-ordinate, we suppose the region $R>a$ with thermal constants $k, \kappa$, to be initially at zero temperature. The region $R<a$ is filled with a perfect conductor initially at temperature $T_{0}$. Conditions being otherwise as before, we have

$$
\left.\begin{array}{rl}
\frac{\partial T}{\partial t} & =\kappa \frac{1}{R} \frac{\partial^{2}(R T)}{\partial R^{2}}+G T, \quad R>a, t>0, \\
T & =0, \quad R>a, t=0, \\
T & =T^{\prime}, \quad R=a, t>0, \\
k \frac{\partial T}{\partial R} & =c^{\prime} \frac{d T^{\prime}}{d t}, \quad R=a, t>0, \\
T^{\prime} & =T_{0}, \quad t=0,
\end{array}\right\}
$$

where $c^{\prime}$ is the heat capacity of the perfect conductor per unit area of the interface. 
For convenience, set $R-a \equiv x, \kappa t \equiv \tau, R T / a T_{0} \equiv u, T^{\prime} / T_{0} \equiv u^{\prime}, \frac{G}{\kappa} \equiv H$ and $k / c^{\prime} \kappa \equiv w$. Then

$$
\left.\begin{array}{rl}
\frac{\partial u}{\partial \tau} & =\frac{\partial^{2} u}{\partial x^{2}}+H u, \\
u & =0, \quad \tau=0, \\
u & =u^{\prime}, \quad x=0, \\
w\left(\frac{\partial u}{\partial x}-\frac{u}{a}\right) & =\frac{d u^{\prime}}{d \tau}, \quad x=0, \\
u^{\prime} & =1, \quad \tau=0 .
\end{array}\right\}
$$

In the same manner as before, we find

$$
\bar{u}=\frac{e^{-\alpha x}}{p+\frac{w}{a}+\alpha w},
$$

so that

$$
u=\frac{1}{2 \pi i} \int_{L} \frac{e^{p \tau-\alpha x}}{p+\frac{w}{a}+\alpha w} d p .
$$

If now $p+\frac{w}{a} \equiv P$, and $H+\frac{w}{a} \equiv K,(32)$ becomes

where

$$
\frac{R T}{a T_{0}} \equiv u=\frac{e^{-\frac{w \tau}{a}}}{2 \pi i} \int_{L} \frac{e^{P \tau-\beta x}}{P+\beta w} d P,
$$

$$
\beta \equiv \sqrt{P-K} \text {. }
$$

Comparison with (5), (6) shows that $T / T_{0}$ is then obtained from the linear solution by multiplying by $\frac{a}{R} e^{-\frac{w \tau}{a}}$ and replacing $x$ by $R-a$ and $H$ by $H+\frac{w}{a}$. Thus, if we set $z \equiv R-a+w \tau$; $h^{2} \equiv \frac{w^{2}}{4}-H-\frac{w}{a},(13)$ yields :

$$
\frac{T}{T_{0}}=\frac{a}{R} \frac{e^{w\left(\frac{z}{2}-\frac{\tau}{a}\right)}}{2 \sqrt{\pi}} \int_{0}^{\tau}\left(\frac{z}{s^{3 / 2}}-\frac{w}{s^{1 / 2}}\right) e^{-h^{2} s-z^{2} / 4 s} d s,
$$

and it can be verified by substitution in (29) that this satisfies the conditions of the problem. Relations corresponding to (15), (16), (21), (22) can be written down in the same way. For example, by (15)

$$
\begin{aligned}
\frac{T}{T_{0}}=\frac{a}{R} e^{-\frac{u \tau}{a}} \cdot \frac{1}{2 h}\{(h- & \left.\frac{w}{2}\right) e^{-z\left(h-\frac{w}{2}\right)} \operatorname{erfc}\left(\frac{z}{2 \sqrt{\tau}}-h \sqrt{\tau}\right) \\
& \left.+\left(h+\frac{w}{2}\right) e^{z\left(h+\frac{w}{2}\right)} \operatorname{erfc}\left(\frac{z}{2 \sqrt{\tau}}+h \sqrt{\tau}\right)\right\},
\end{aligned}
$$

provided $h^{2} \equiv \frac{w^{2}}{4}-H-\frac{w}{a}>0 . \quad$ Otherwise, (35) may be used.

(35), (36) include, when $H=0$, the interesting case of a perfectly conducting sphere heated or cooled in an infinite medium. The solution, curiously enough, does not seem to have been given previously. 
Again, for large $(R-a) / 2 \sqrt{\tau},(21)$ becomes

$$
T / T_{0}=\frac{a}{R} e^{\left(H+\frac{w}{a}\right) \tau}\left[\operatorname{erfc} \frac{R-a}{2 \sqrt{\tau}}+\sum_{1}^{\infty} B_{n}(2 \sqrt{\tau})^{n} i^{n} \operatorname{erfc} \frac{R-a}{2 \sqrt{\tau}}\right],
$$

where $B_{n}$ is defined by (20) with $H$ replaced by $H+\frac{w}{a}$. Similarly, by (22),

$$
T^{\prime} / T_{0}=e^{\left(H+\frac{w}{a}\right) \tau}\left[1-2 w \sqrt{\frac{\tau}{\pi}}+\left(w^{2}-\frac{w}{a}-H\right) \tau+\ldots\right] .
$$

The remarks concerning the variation of $T^{\prime}$ in the linear case apply equally to the spherical problem. We may illustrate the behaviour of $T^{\prime}$ by a special case, corresponding now to $h=0$, that is, to $\frac{w^{2}}{4}-\frac{w}{a}=H$. From (36), by letting $h$ approach zero we deduce

$$
T^{\prime} / T_{0}=e^{\xi^{2}}\left(1-\frac{4}{a w}\right)\left[\left(1+2 \xi^{2}\right) e^{\xi^{2}} \operatorname{erfc} \xi-\frac{2 \xi}{\sqrt{\pi}}\right]
$$

where, as before, $\xi \equiv w \sqrt{\tau} / 2$. As would be expected, (39) reduces to (23) when the radius $a \rightarrow \infty$.

Since $a w=\frac{3 C \rho}{C^{\prime} \rho^{\prime}}$, where $C, \rho$ are the specific heat and density of the medium $(R>a)$ and $C^{\prime}, \rho^{\prime}$ corresponding quantities for the source $(R<a)$, and since we require aw $>4$, (39) cannot apply to any solid sphere in a gas : for such systems, $a w \sim 10^{-2}$. However, it is possible for $a w$ to exceed 4 in the case of a liquid or solid external medium. For example, aluminium spheres in nitroglycerin would give $a w \sim 5$. If we take this value for illustration, (39) has a minimum at $\xi_{c} \sim 2 \cdot 4$, so that $w \sim 5 / \sqrt{\tau_{c}}$. Adopting a typical value of $0.01 \mathrm{~cm} .2 / \mathrm{sec}$. for $\kappa$ and requiring that $t_{c} \ngtr 1$ sec. for explosion, we conclude $w \sim 50 \mathrm{~cm} .^{-1}$, whereupon $a \sim 1 \mathrm{~mm}$. Then $H \sim 125$, and so $G \equiv \kappa H \sim 1 \cdot 25$. However (3), for nitroglycerin, $G \sim 10^{17} T_{0}{ }^{-1} e^{-20,000 / T_{0}}$ by equation (26). Hence, finally, $T_{0} \sim 600^{\circ} \mathrm{K}$. The analysis therefore suggests that an aluminium sphere of radius $1 \mathrm{~mm}$. will ignite nitroglycerin if raised to a temperature of about $300^{\circ} \mathrm{C}$. The result is quite reasonable, since nitroglycerin in bulk ignites at about $200^{\circ} \mathrm{C}$.

\section{REFERENCES}

(1) W. Horenstein, Quart. Appl. Math., 3, 183 (1945).

(2) D. H. Hartree, Mem. and Proc. Manchester Lit. and Phil. Soc., 80, 85 (1935).

(3) L. Phillips, Nature, 160, 753 (1947).

Imperial Chemical Industries Ltd.

Nobel Division, Research Department

Stevenston, Ayrshire 